\title{
Postharvest Highbush Blueberry Fruit Antimicrobial Volatile Profiles in Relation to Anthracnose Fruit Rot Resistance
}

\author{
James J. Polashock ${ }^{\mathbf{1}}$ \\ Fruit Laboratory, Agricultural Research Service, U.S. Department of Agriculture, 125A Lake Oswego \\ Road, Chatsworth, NJ 08019
}

\author{
Robert A. Saftner \\ Produce Quality and Safety Laboratory, Henry A. Wallace Agricultural Research Center, \\ Agricultural Research Service, U.S. Department of Agriculture, Beltsville, MD 20705 \\ Matthew Kramer \\ Biometrical Consulting Service, Henry A. Wallace Agricultural Research Center, Agricultural \\ Research Service, U.S. Department of Agriculture, Beltsville, MD 20705
}

\begin{abstract}
AdDitional INDEX words. aldehyde, antifungal, Colletotrichum acutatum, terpene, Vaccinium corymbosum
ABstract. Fruit of highbush blueberry (Vaccinium corymbosum L.) produce antimicrobial volatiles, including trans2-hexenal, that may confer resistance to anthracnose fruit rot, an important postharvest disease caused by Colletotrichum acutatum J.H. Simmonds. To investigate whether aromatic volatiles in highbush blueberry fruit are associated with postharvest fruit rot resistance, we compared volatiles emitted from whole fruit and extracts from fruit kept in air at $20^{\circ} \mathrm{C}$ for 0 to 6 days postharvest from cultivars having a wide range of resistance to anthracnose. Antimicrobial volatiles detected included the aldehydes, trans-2-hexenal and hexanal; the monoterpenes, limonene, linalool, 8-hydroxylinalool, $\alpha$-terpineol, and terpinyl acetate; and the sesquiterpenes, cadinene, caryophyllene, and $\alpha$-farnesene. There were significant correlations between some detected volatiles and these differed in whole fruit and extracts. Hexanal (in fruit extracts), trans-2-hexenal, terpinyl acetate, and cadinene emissions increased in most cultivars when fruit were kept in air at $20^{\circ} \mathrm{C}$ for various times postharvest. Volatile emissions from whole fruit and extracts varied widely among the cultivars with early ripening cultivars generally showing higher volatile emissions than later ripening cultivars. Although the cultivars tested differed in quantities, and in some cases, the types of volatiles produced, these differences were not related to pedigree (i.e., species composition) nor to known anthracnose resistance ratings. Except for the confounded emissions of terpinyl acetate and cadinene, more than $80 \%$ of the variation observed for each volatile was attributable to the cultivar (genetic), year (environmental), and cultivar-byyear interaction. The results suggest that, although antimicrobial aldehydes and terpenes emitted from fully ripe highbush blueberry fruit and extracts might be important flavor and aroma components, they do not significantly contribute to disease resistance against anthracnose fruit rot.
\end{abstract}

Much of the highbush blueberry crop goes to fresh market and postharvest decay is an ongoing problem. The most important postharvest disease of blueberry is anthracnose fruit rot [causal agent Colletotrichum acutatum and, in some growing regions, C. gloeosporioides (Penz.) Penz. and Sacc.]. Losses resulting from anthracnose preharvest are typically $3 \%$ to $5 \%$, and postharvest losses can reach $100 \%$ (Milholland, 1995).

Highbush blueberry fruit typically becomes infected in the field while still green. Spores germinate on infected fruit, form appressoria, and then become dormant until the fruit ripens (Daykin and Milholland, 1984). Highbush blueberry cultivars have shown a wide range of susceptibility to this disease (Polashock et al., 2005) and the mechanism of disease resistance among cultivars is not known.

Aromatic volatiles contribute to the aroma and flavor of fruit and vegetables, including highbush blueberry (Forney, 2001).

Received for publication 30 Jan. 2007. Accepted for publication 3 Aug. 2007. We thank Kristia Adams, Willard Douglas, and Allan Stretch for technical assistance. We also thank Nicholi Vorsa for sharing unpublished data.

${ }^{1}$ Corresponding author. E-mail: james.polashock@ars.usda.gov.
Emitted volatiles from highbush blueberry fruit are primarily aldehydes and terpenes (Baloga et al., 1995; Overton and Manura, 1999). Aldehydes, organic compounds containing a terminal carbonyl (-CHO) group, are often derived from lipid peroxidation (Latrasse, 1991). Terpenes, primarily plant hydrocarbons formed from linked isoprene $\left(\mathrm{C}_{5} \mathrm{H}_{8}\right)$ units, are derived from the mevalonate and methylerythritol pathways and specific terpene synthases (Dudareva et al., 2004). These and other volatiles emitted from highbush blueberry and other fresh produce are active against bacteria, fungi, and viruses (Cowan, 1999; Dorman and Deans, 2000; Gardini et al., 2001; Utama et al., 2002). The incorporation of high concentrations of various antimicrobial volatiles, including hexanal and trans2-hexenal, into the storage atmosphere of fresh produce has been shown to reduce postharvest decay (Corbo et al., 2000; Lanciotti et al., 1999). Most, if not all, terpenes emitted from highbush blueberry have antifungal activities and include 1,8cineole (Tabanca et al., 2006; Wilson et al., 1997), caryophyllene (Raintree Nutrition Inc., 2004), cadinene (Song et al., 2007), $\alpha$-farnesene (Govinden-Soulange et al., 2004), 2- and 3carene (Archbold et al., 1997; Cavaleiro et al., 2006; Himejima et al., 1992), limonene (Archbold et al., 1997; Himejima et al., 
1992; Wilson et al., 1997), linalool (Carson and Riley, 1995; Fraternale et al., 2004; Plotto et al., 2003), terpinolene (Himejima et al., 1992), $\alpha$-terpineol (Carson and Riley, 1995; Tepe et al., 2004), and $\alpha$-terpinyl acetate (Peana et al., 1999). 8Hydroxylinalool, another volatile emitted from highbush blueberry fruit, may have antifungal activity because oxygenated terpenes generally have more antifungal activity than nonoxygenated ones (Caccioni and Guizzardi, 1994; Knobloch et al., 1989). In addition, several essential oils with antifungal activities have as their major components the same terpenes as those emitted from highbush blueberry (Al-Howiriny, 2003; Sipailiene et al., 2006; Wilson et al., 1997). A few essential oils have been tested and shown to have specific antifungal activity against Colletotrichum Corda in Strum. species, including $C$. acutatum (Demirci et al., 2006; Tabanca et al., 2006, 2007).

Preliminary analysis of highbush blueberry volatiles showed that cultivars 'Duke' and 'Bluecrop' had similar volatile profiles, but 'Duke' had higher concentrations of emitted antimicrobial volatiles (N. Vorsa, personal communication). These two cultivars were shown to have significant differences in anthracnose fruit rot resistance with 'Duke' being more resistant (Polashock et al., 2005). In addition to direct antimicrobial activities, trans-2-hexenal and other emitted volatiles may induce expression of host defense-related genes (Bate and Rothstein, 1998; Ding et al., 2002).

Based on the known presence of these antimicrobial volatiles in highbush blueberry and the differences in volatile concentrations of 'Duke' and 'Bluecrop', we hypothesized that volatiles naturally produced in highbush blueberry reduce postharvest decay caused by $C$. acutatum. To test this hypothesis, 10 (in 2004) and six (in 2005) highbush blueberry cultivars spanning the range from very susceptible to very resistant were surveyed for qualitative and quantitative differences in fruit volatile emissions when the fruit were harvested and kept in air at $20{ }^{\circ} \mathrm{C}$ for 0 to $6 \mathrm{~d}$ (to simulate market shelf conditions). Because many of the antimicrobial volatiles are produced when plant tissues are wounded (Chappell, 1995; Hatanaka, 1993; Mari and Guizzardi, 1998), we also measured volatile emissions from whole fruit inoculated with anthracnose (i.e., pathogen "wounded" as fungal hyphae penetrate the fruit surface) and from fruit extracts prepared from both noninoculated and C. acutatum-inoculated fruit.

Primary objectives were to: 1 ) determine the quantitative and qualitative differences in fruit volatile emissions across representative highbush blueberry cultivars with varying susceptibility to anthracnose fruit rot, 2) determine the qualitative and quantitative changes in fruit volatile emissions when fruit were kept in air at $20^{\circ} \mathrm{C}$ for varying times postharvest, and 3 ) investigate if known fruit rot susceptibilities are determined by differences in fruit volatile emissions. Secondary goals were to 1) determine if volatiles, especially those known to be antimicrobial, could be induced by inoculation with anthracnose spores; 2) determine what genetic, environmental, and other factors affected fruit volatile emissions and to what degree; and 3) characterize correlations among volatiles.

\section{Materials and Methods}

Plant material. All highbush blueberry cultivars used in this study were mature field-grown plants maintained at the P.E. Marucci Center for Blueberry and Cranberry Research, Chatsworth, NJ. In 2004, 10 cultivars were selected based on anthracnose fruit rot susceptibility (Polashock et al., 2005), availability, and fruit-ripening season. Six of the same 10 cultivars were retested in 2005. The cultivars and selected characteristics of each are listed in Table 1.

Anthracnose inOCulation and fRUit rot assessment. Colletotrichum acutatum mass-isolates (i.e., a mixture of strains) were collected from infected highbush blueberry shoots and cultured on V8 agar. Anthracnose spores (conidia) were washed from 7 - to 10-d-old cultures grown on V8 agar (without $\mathrm{CaCO}_{3}$ ) plates and diluted in water to $1 \times 10^{6} / \mathrm{mL}$. Clusters of fruit that were just beginning to ripen were sprayed in the field to runoff using a handheld pump sprayer. Clusters were then immediately covered with a plastic bag containing a small strip of moist cheesecloth to maintain high humidity. To reduce heat accumulation in the bags, inoculations were done late in the day and the plastic bags were covered with white paper bags. Noninoculated fruit clusters were treated identically to the inoculated clusters but were sprayed with water only. Bags were removed from all clusters early the next morning. The effects of fungicides on volatile production and emission are not known and therefore fungicides were deliberately withheld from plants used in this study.

To assess levels of anthracnose fruit rot, a $25-\mathrm{g}$ sample of berries collected from each plant of noninoculated and inoculated fruit of each cultivar was incubated in a plastic container lined with moist paper towels. After $5 \mathrm{~d}$ in air at $20^{\circ} \mathrm{C}$, each replicate was scored for the presence of orange spore masses on the fruit surface as an indicator of anthracnose fruit rot. Note that in addition to our measure of sample fruit rot, which is on a plant-by-plant basis and was used in modeling volatile emissions, we also have a measure of historical fruit rot (from previous work and given in Table 1), here provided as an overall cultivar mean. Because plant-to-plant and year-to-year variability can and often does occur, sample fruit rot could differ from the historic cultivar mean fruit rot.

Fruit Collection and SAMPle PReParation. Fully ripe noninoculated and C. acutatum-inoculated fruit were separately

Table 1. Highbush blueberry cultivars and selected characteristics of each.

\begin{tabular}{lccccc}
\hline & Fruit & Ripening & \multicolumn{4}{c}{ Ancestry $(\%)^{\mathrm{w}}$} \\
\cline { 4 - 6 } Cultivar $^{\mathrm{z}}$ & rot $(\%)^{\mathrm{y}}$ & season $^{\mathrm{x}}$ & V. corym. & V. angust. & V. darrowi \\
\hline Berkeley & 45 & Mid & 100 & & \\
Bluecrop $^{2}$ & 78 & Mid & 94 & 6 & \\
Bluegold & 54 & Mid & 75 & 25 & \\
Blueray & 70 & Mid & 94 & 6 & \\
Duke $^{2}$ & 44 & Early & 96 & 4 & 25 \\
Elliott $^{2}$ & 15 & Late & 100 & & \\
June $^{2}$ & 78 & Early & 75 & 25 & \\
Lateblue $^{2}$ & 55 & Late & 100 & & \\
Legacy $^{2}$ & 10 & Late & 73 & 2 & \\
Spartan $^{n}$ & 78 & Early & 94 & 6
\end{tabular}

zThose cultivars used in both 2004 and 2005 are noted by a “2”. ${ }^{\mathrm{y}}$ Fruit rot expressed as percent infected berries, from Polashock et al. (2005).

${ }^{\mathrm{x}}$ Ripening time relative to other highbush blueberry cultivars.

wheoretical species composition based on pedigree information $(V$. corym.$=$ Vaccinium corymbosum L., V. angust. $=$ Vaccinium angustifolium Aiton, $V$. darrowi $=$ Vaccinium darrowi Camp). Note that the ancestry of 'Bluegold' is not definitive because the species composition of one of the parents in the pedigree (i.e., Ashworth) is unclear. 
collected from three plants (each plant is considered a replicate) of each cultivar and any decayed or otherwise damaged fruit removed. From each replicate collection of noninoculated or inoculated berries, three 25 -g samples were placed into paper bags, each kept for 0,2 , or $4 \mathrm{~d}$ at $20^{\circ} \mathrm{C}$, and then placed in a $-20{ }^{\circ} \mathrm{C}$ freezer. Within $3 \mathrm{~d}$, frozen fruit samples were individually ground to a slurry with a cold mortar and pestle. One milliliter of each extract was transferred to a 4-mL glass vial containing $0.3 \mathrm{~mL}$ of $3 \mathrm{M} \mathrm{CaCl}_{2}$ to inhibit enzymatic activities associated with volatile biosynthesis (Buttery and Ling, 1993), capped with a Teflon-lined septum, and stored up to 3 weeks at $-80{ }^{\circ} \mathrm{C}$ before volatile collection and analysis.

Whole berry samples $(25 \mathrm{~g})$ from each replicate collection were shipped overnight in foam coolers to Beltsville, MD, and then transferred to $473-\mathrm{cm}^{3}$ canning jars and kept for $6 \mathrm{~d}$ at $20{ }^{\circ} \mathrm{C}$. At 1 to $2 \mathrm{~d}$ and again at 5 to $6 \mathrm{~d}$, each canning jar was sealed for 24-h collections of volatiles being emitted from the whole fruit (see next section for details).

Volatile COLLECTION AND ANALYSIS. In preparation for volatile sampling, frozen fruit extracts were thawed and equilibrated for $5 \mathrm{~min}$ at $20^{\circ} \mathrm{C}$, and canning jars containing whole fruit were equilibrated at $20^{\circ} \mathrm{C}$ for greater than $4 \mathrm{~h}$ before sealing each jar with a metal lid containing a rubber gasket and a 5-mm-diameter rubber septum for sample access. A solid-phase microextraction (SPME; Suppelco Co., Bellefonte, PA) technique for collection and gas chromatography (GC) analysis of aromatic volatiles was performed as previously described (Saftner, 1999). Briefly, a SPME fiber (1 cm long, $100 \mu \mathrm{m}$ thick) coated with polydimethylsiloxane was used to collect volatiles emitted from fruit extracts and whole fruit by virtue of its sorption characteristics (Pawliszyn, 1997). The SPME device consisted of a retractable fiber enclosed in a metal needle. During sampling, after entering the $4-\mathrm{mL}$ vial or $473-\mathrm{cm}^{3}$ canning jar, the fiber was manually extended, volatile sorption occurred for $16 \mathrm{~min}$ (fruit extracts) or $24 \mathrm{~h}$ (whole fruit), then the fiber was retracted before removal from the sampling container. The sorbed volatiles were desorbed from the fiber for $2 \mathrm{~min}$ at $250{ }^{\circ} \mathrm{C}$ into a glass-lined, splitless injection port of a gas chromatograph (model 6890; Hewlett Packard Co., Rockville, MD) equipped with a flame ionization detector, which was used to measure relative volatile gas concentrations. Construction of calibration curves for each volatile analyte in each highbush blueberry sample is not feasible; thus, volatile concentrations are reported in detector response units of picoamps rather than absolute amounts (Saftner et al., 2002). For volatile identification, a GC/mass spectrometer procedure was used (Saftner et al., 2002). Identification of volatile analytes was confirmed by comparison of collected mass spectra with those of standards and spectra in the National Institute for Standards and Technology mass spectral library, Search Version D.04.00 (Agilent Technologies, Rockville, MD).

Statistical anAlyses. The basic design of the experiment was factorial (all main effects are crossed so that all combinations are present) with cultivars, treatment (inoculated or noninoculated), and holding time as the main effects. Data for the analysis of extracts were collected in 2 consecutive years; in some, the analyses were conducted separately for each year; in others, year effects were included in the modeling (see subsequently). Whole fruit were only assayed in 2005. Significance for all statistical tests was set at $P<0.05$.

For each cultivar-treatment combination, the berries from each of three plants (each plant was considered a replicate) were divided into three samples per plant, and extracts were prepared as detailed previously at each of the three holding times $(0,2$, or $4 \mathrm{~d})$. Similarly, whole fruit from each of three plants were separately divided into two samples per plant and each sample was analyzed at one of the two holding times ( 1 to $2 \mathrm{~d}$ or 5 to $6 \mathrm{~d}$ ). Dividing the berries from each plant like this introduces a blocking effect. Because each group of three samples comes from the same plant, this was accounted for in the analysis by including a within-plant random effect to capture the correlation induced by subsampling from an individual plant.

A preliminary analysis found that, for the area under the curve for these volatiles, the variance depended on the mean (i.e., larger means were accompanied by larger standard errors). We transformed the data for the analyses using $y=\log (x+1)$, where $x$ is the area under the curve for the volatile. This transformation removed this dependency and appeared to be a good choice for all volatiles. Below we outline the four types of statistical analyses performed to address our objectives: 1) correlation between an indicator of resistance (historic percent fruit rot) and detected volatile concentrations, 2) stepwise multiple regression of the transformed volatile data to determine which independent variables were useful predictors of the volatile data, with specific interest in the relationship between volatiles and susceptibility to fruit rot, and to assess the effects of postharvest holding time and inoculation treatment, 3) a variance decomposition to determine what proportion of the explained variance in the volatiles was captured by each of the independent variables, and 4) lagged correlations among volatiles, because there are known pathways between at least some volatiles that might be influenced by postharvest holding time.

We estimated Pearson correlations between percent fruit rot (Table 1) determined from an earlier study with large sample sizes (Polashock et al., 2005) and the mean of the transformed volatile data grouped by treatment (inoculated versus noninoculated), holding time, and year. Strong correlations would suggest that genetic differences in rot resistance are associated with differing concentrations of one or more volatiles, and one might then use volatile concentrations to predict rot resistance.

Blocking by plant in the stepwise regression analyses introduced a random effect. We used software developed by one of us (Kramer, 2004), which does automatic model selection in the mixed models framework. The program is written in Perl, calls Proc Mixed in SAS (SAS Institute, Cary, NC) for REML model estimation and uses Akaike's information criterion (Burnham and Anderson, 1998) to determine model improvement. Quantitative independent variables were standardized (centered on zero and scaled so that their standard deviation equals one) before model selection.

Cultivar type was not used as a candidate independent variable in the stepwise multiple regression because ripening time was available only as a cultivar mean (ripening time could have no explanatory power if cultivar type was also in the model). Model selection was performed separately for the 2004 (10 cultivars) and 2005 (six cultivars) data sets for fruit extracts and for the 2005 data set of whole fruit. Postharvest holding time and treatment (inoculated or noninoculated), whether significant or not, were forced into each model. Candidate additional independent variables were plant-specific estimate of percent fruit rot, two measures of ripening day (one, a cultivar average for the date when $50 \%$ of the fruit was ripe, the other the date that fruit for this study was harvested), and all 
two-way interactions. Only cultivars that had at least one sample with a positive measurement for the volatile were used in the model selection process. This avoided, for some volatiles, forcing the model to fit a large number of zeroes. Thus, models for some volatiles were based on different cultivars than models for others. We also calculated an $\mathrm{R}^{2}$ based on maximum likelihood statistics (Kramer, 2005) for the final models.

SAS Proc Mixed was used to estimate variances for the variance decomposition by considering all factors as random effects and estimating the contribution to the total variance of each. We included cultivar as a factor to estimate the genetic contribution (and so did not include ripening time and percent fruit rot; the latter tends to be cultivar-specific). Because we wanted to estimate the variance attributable to year (a proxy for environmental effects), only the extract data for the six cultivars common to both years were used. Thus, both the data and the models differed from those in the stepwise regression analysis.

Pearson correlations and scatterplots were used to investigate the correlations among time-lagged volatiles with the 2 years of extracts analyzed separately. Means of the three (or two, in the case of fruit extracts) samples were used in this analysis to represent a cultivar. Because not all volatiles were present in each cultivar, correlations between two volatiles were calculated only if at least three cultivar means were available. Because sample sizes for each calculated correlation were small (a maximum of 10 and minimum of three), correlations must be high to be declared significant.

\section{Results}

VOLATILES EMITTED FROM WHOLE FRUIT AND FRUIT EXTRACTS OF SIX HIGHBUSH BLUEBERRY CULTIVARS. The cultivars tested exhibited dramatic differences in both the specific volatiles emitted and the quantities of the volatiles in common among the cultivars. A typical gas chromatogram of volatiles from a highbush blueberry fruit extract is shown in Figure 1. The

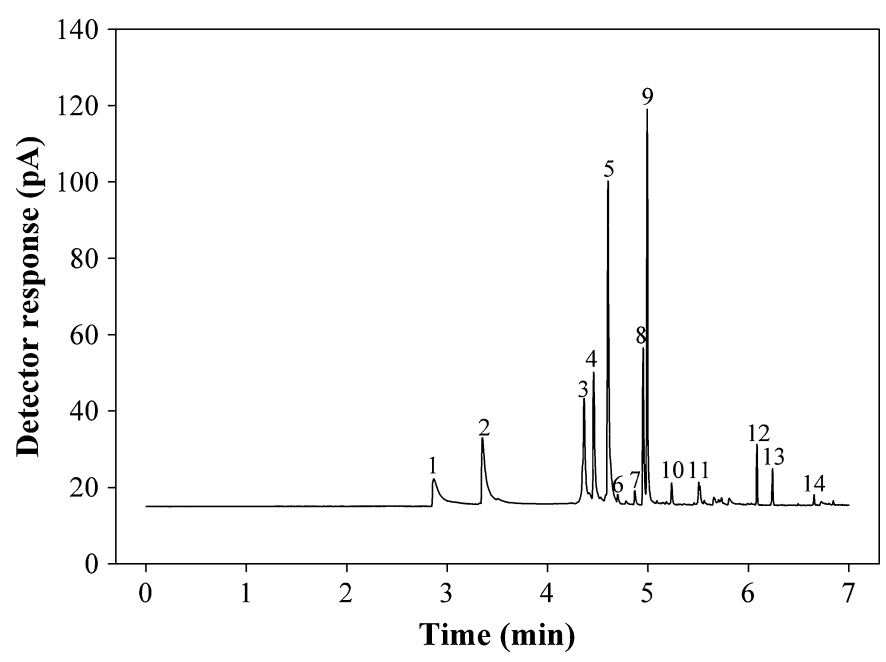

Fig. 1. Representative gas chromatogram of solid-phase microextractioncollected volatiles emitted from 'Duke' highbush blueberry fruit extract. Identified volatiles are as follows: $1=$ hexanal; $2=$ trans-2-hexenal; $3=8$ hydroxylinalool; $4=3$-carene; $5=$ limonene; $6=1,8$-cineole; $7=$ terpinolene; $8=\alpha$-terpinene; $9=$ linalool; $10=$ column bleed material; $11=\alpha$-terpineol; 12 = contaminant plasticizer; $13=$ terpinyl acetate and cadinene; $14=$ caryophyllene. Most other peaks (less than $10 \%$ of total emitted volatiles) corresponded to esters and alkanes having no known antimicrobial activities. volatiles identified fell into four classes, aldehydes (hexanal and trans-2-hexenal), terpenes, esters, and alkanes. Volatiles detected from both whole fruit and fruit extracts with potential antimicrobial activity included the aldehyde, trans-2-hexenal; the monoterpenes, $\alpha$-terpineol, terpinyl acetate, limonene, and linalool; and the sesquiterpenes, cadinene and caryophyllene. Whole fruit, but not fruit extracts, also emitted the sesquiterpene, $\alpha$-farnesene; conversely fruit extracts, but not whole fruit, emitted the aldehyde, hexanal, and the monoterpene, 8-hydroxylinalool. A few cultivars, especially 'Duke', emitted the monoterpenes, terpinolene and $\alpha$-terpinene, from both whole fruit and fruit extracts. Small quantities of the monoterpenes, 3-carene and 1,8-cineole, were rarely detected and only in fruit extracts. Figure 2 gives the relative volatile concentration means for the six cultivars used in both years and their standard errors (based on two yearly means, thus reflecting year-to-year variability) for fruit extracts and concentration means only for whole fruit for 2005. The small plant-to-plant variability (see subsequently) is not depicted.

Anthracnose fruit Rot. Anthracnose levels, as measured in a subset of each fruit sample, were very high in the inoculated fruit with some cultivars reaching $100 \%$ (Fig. 3). Levels in the controls (noninoculated) were generally low in 2004 but high in 2005 (Fig. 3). The strong effect of inoculation on percent fruit rot in 2004 appears to have had no apparent effect on subsequent volatile emissions of fruit sampled from these inoculated plants.

Modeling volatile Emissions. Summary results of the automatic model selections for extracts and whole fruit are provided in Table 2 . All effects selected to be in the models were significant at $P \leq 0.01$. The design effects, holding time and treatment, were forced into all models. Holding time did affect some volatiles, more so for extracts than whole fruit (see subsequently). The treatment main effect was never significant nor was an interaction involving treatment ever selected. This indicates that inoculation with the pathogen had a negligible effect on production of volatiles by the fruit. Most $\mathrm{R}^{2}$ values were relatively high, giving us confidence that we captured most of the important independent variables affecting volatile emissions. $\mathrm{R}^{2}$ statistics were generally lower for whole fruit than extracts and lower for 2004 fruit extracts than 2005 fruit extracts (probably because the reduced number of cultivars in 2005 had fewer disparate points for the model to try to fit).

ALDEHYDE CONCENTRATIONS EMITTED FROM WHOLE FRUIT AND FRUIT EXTRACTS. During holding at $20{ }^{\circ} \mathrm{C}$, emission of trans2-hexenal increased from whole fruit of all cultivars (Fig. 2). We found mild evidence of a relationship between aldehyde emissions of fruit extracts and historical levels of fruit rot. For the 0 -d holding time in 2004 (with 10 cultivars), and for both inoculated and control groups, there was a significant positive correlation (which is the opposite of our prediction that increased volatiles would decrease fruit rot) for both aldehydes (results not shown). There was no statistically significant relationship in 2005 (with six cultivars); however, all correlation coefficients were estimated to be positive. Whole fruit emissions were also not significant with correlation estimates close to zero.

For fruit extracts, the trans-2-hexenal concentration remained stable or increased, depending on cultivar, as the fruit holding time increased (Fig. 2). This pattern of generally increasing trans-2-hexenal emission from extracts during holding was similar to the pattern observed in whole fruit (Fig. 2). 


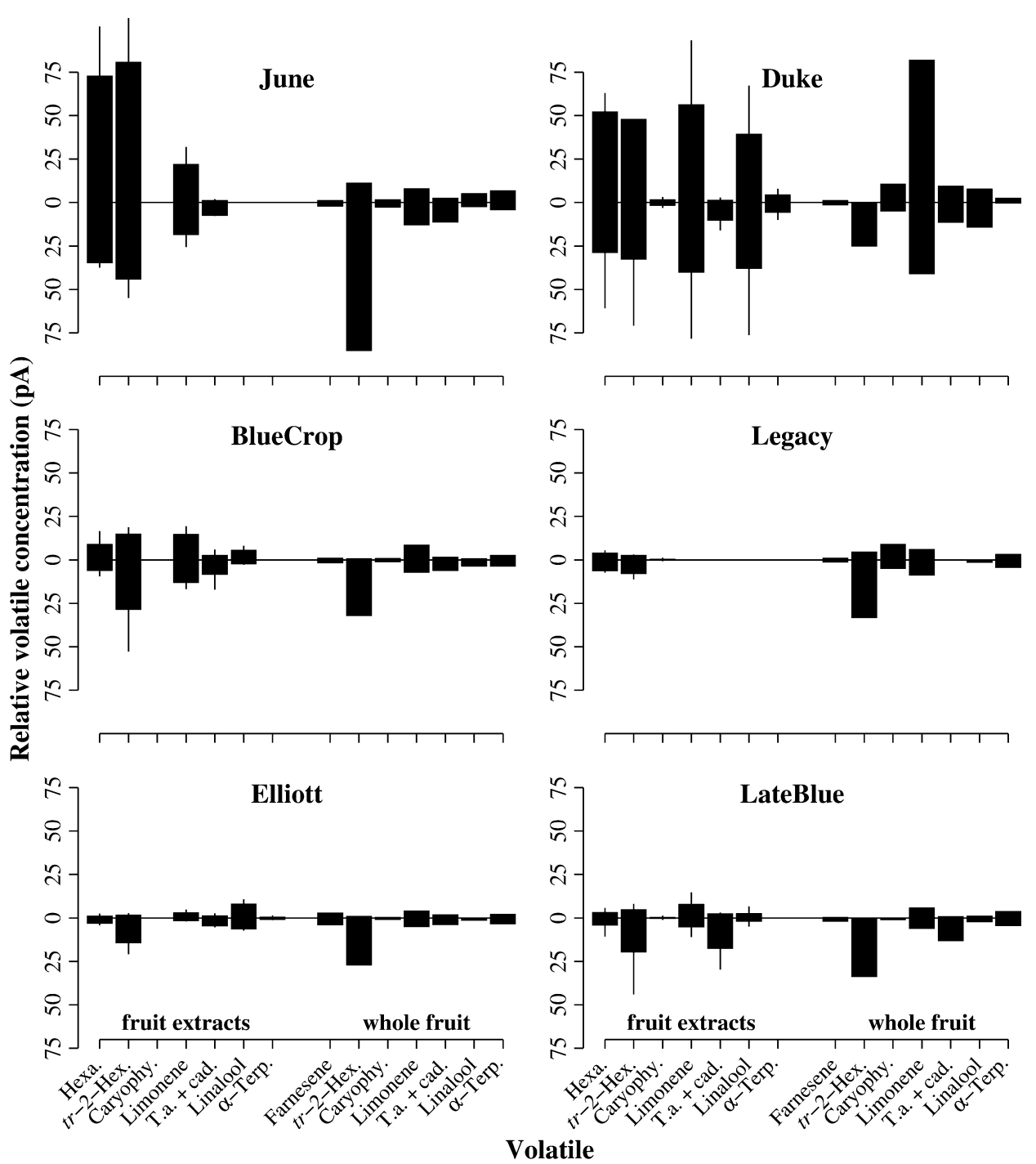

Fig. 2. Relative volatile concentrations in fruit extracts and whole fruit of highbush blueberry cultivars 'June', 'Duke', 'Bluecrop', 'Legacy', 'Elliott', and 'Lateblue'. Bars going up represent data from the first time point ( $0 \mathrm{~d}$ for extracts and 1 to $2 \mathrm{~d}$ collection for whole fruit). Bars going down represent data from the last time point ( $4 \mathrm{~d}$ for extracts and 5 to $6 \mathrm{~d}$ for whole fruit). Volatile abbreviations are: Hexa. $=$ hexanal, tr-2-Hex. $=$ trans2-hexenal, caryophy. $=$ caryophyllene, T.a. + cad. $=$ Terpinyl acetate + cadinene, $\alpha$-Terp. $=\alpha$-Terpineol.

terpene concentrations from whole fruit or from fruit extracts were correlated with historic levels of fruit rot (results not shown); thus, volatile concentration data among cultivars did not predict cultivar resistance to rot.

Emission of oxygenated (linalool) and acetylated (terpinyl acetate) monterpenes and sesquiterpenes (cadinene and $\alpha$-farnesene) from whole fruit generally increased with holding time of whole fruit (Fig. 2) with the exception of the early ripening cultivars, 'June' (linalool decreased) and 'Duke' (terpinyl acetate decreased). Neither terpinyl acetate nor cadinene emissions were detected in 'Legacy' fruit. Cadinene was only detected after $5 \mathrm{~d}$ holding time, although not for all cultivars; thus, its emission was induced postharvest.

For whole fruit, the model selection procedure found only a holding time by sample fruit rot interaction for $\alpha$-farnesene and terpinyl acetate + cadinene and a holding time by harvest day interaction for linalool. Coefficients, except for terpinyl acetate + cadinene at the 5-d holding time, were negative; lower concentrations of these volatiles were emitted with increases in sample percent rot or with later harvest days, and these relationships differed significantly for the two holding times. $\mathrm{R}^{2}$ values for these models were $\approx 0.5$, suggesting that additional explanatory variables were needed.

$\alpha$-Terpineol, limonene, and caryophyllene emissions during holding were variable in pattern. Limonene

Similar to results with historical fruit means, trans-2hexenal emission from extracts was, in part, predicted by the percent fruit rot of individual samples, although the relationship differed by year and holding time (Table 2). Like historical fruit rot means, the relationships were generally positive (more so in 2004). Hexanal was not detected evolving from whole fruit and there was no detectable relationship between trans-2-hexenal and sample fruit rot in whole fruit.

Both aldehydes (indeed all volatiles except $\alpha$-terpineol) showed effects resulting from postharvest holding time either as a main effect or involved in an interaction with percent fruit rot for at least one of the years. Holding time effects were stronger for fruit extracts than for whole fruit. Harvest day was the only other independent variable selected for these models and appears to have an inverse relationship with volatile emissions. Cultivars with later fruit ripening times produced lower aldehyde emissions (Fig. 2).

TERPENE CONCENTRATIONS EMITTED FROM WHOLE FRUIT AND FRUIT EXTRACTS. Among the cultivars tested, none of the emitted emissions increased in 'June', decreased in 'Duke', and did not change in the other cultivars during holding. Emissions of oxygenated $\alpha$-terpineol decreased in 'June' and 'Duke' while increasing in the other cultivars during holding. Caryophyllene emission increased in 'June', 'Bluecrop', and 'Lateblue'; decreased in 'Duke' and 'Legacy'; and remained stable in 'Elliott' during holding.

The model selection procedure for whole fruit found no explanatory variables for $\alpha$-terpineol $\left(\mathrm{R}^{2}\right.$ near 0$)$, and found harvest day, with a negative coefficient, for caryophyllene and limonene. For caryophyllene, this was offset by a positive coefficient for the average $50 \%$ ripe date, in effect allowing those cultivars in which the two dates differ substantially to have different relationships between harvest date and caryophyllene concentrations from these relationships in the other cultivars. Caryophyllene concentrations also increased with decreasing sample percent rot. The model for this terpene had a high $\mathrm{R}^{2}$ of 0.81 .

Headspace concentrations of most terpenes (limonene, linalool, $\alpha$-terpineol, $\alpha$-terpinene, terpinolene) from fruit 


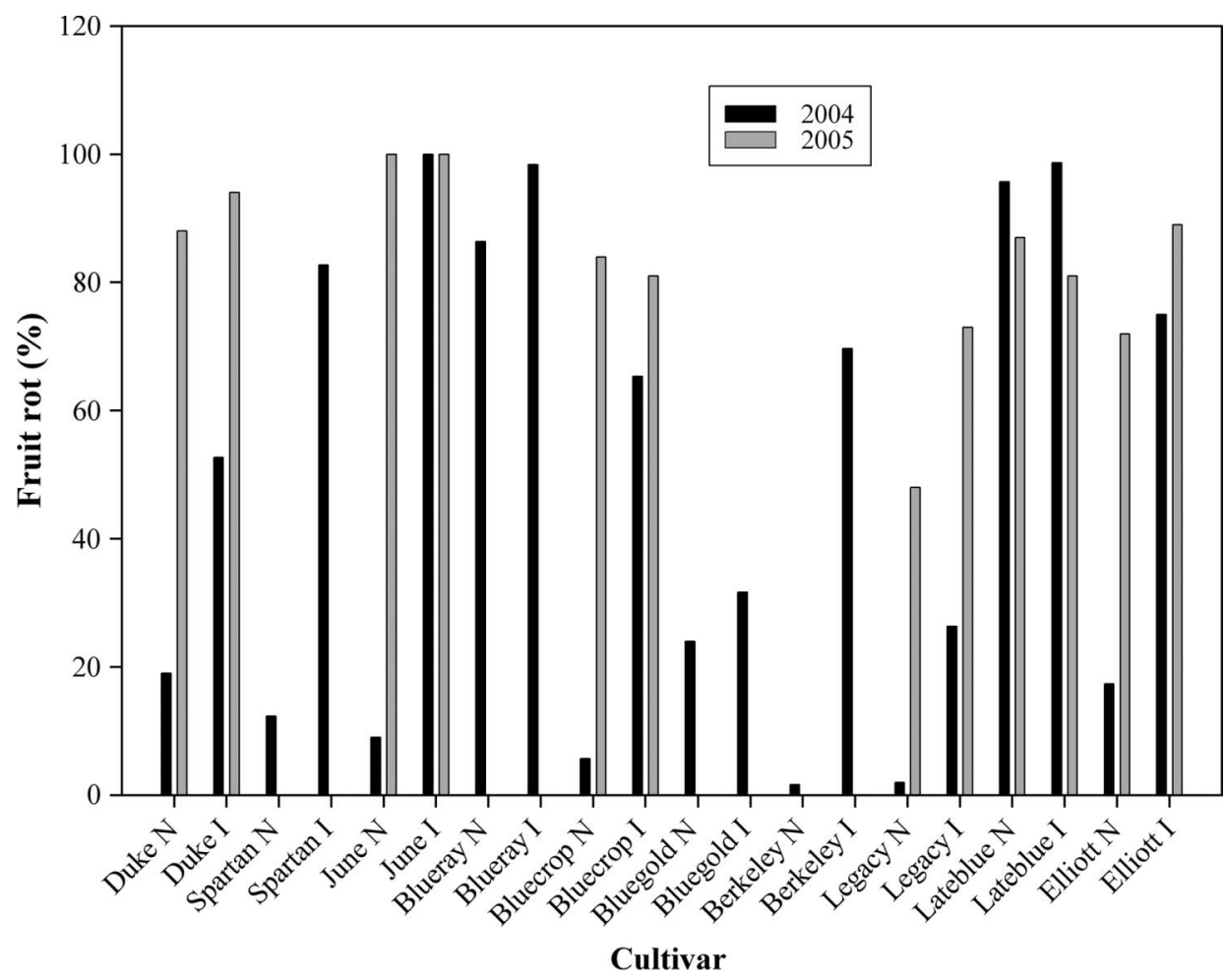

Fig. 3. Anthracnose levels in highbush blueberry after a 5-d postharvest incubation at $20^{\circ} \mathrm{C}$ for noninoculated (N) and inoculated (I) fruit.

extracts prepared from fruit kept 0 or $4 \mathrm{~d}$ generally remained stable or decreased as fruit holding time increased (Fig. 2). However, headspace concentration of terpinyl acetate + cadinene from fruit extracts increased as fruit holding time increased. Compared with terpene emission patterns from whole fruit during holding, fruit extracts emitted relatively low concentrations of linalool after holding and had no detectable $\alpha$-farnesene.

Models for all terpenes for fruit extracts, other than $\alpha$ terpineol, had significant holding time effects (caryophyllene only for 2005 when it was involved in an interaction with sample percent rot). Sample percent rot was a significant explanatory variable for all terpenes except limonene and terpinyl acetate + cadinene with a positive coefficient unless harvest day was also in the model. Other effects were rare and did not appear in both years. $\mathrm{R}^{2}$ values for terpenes were higher for 2005 than for 2004 models (Table 2).

VARIANCE DECOMPOSITION. Results from the variance decomposition (Table 3) are consistent with those from the automatic model selection, although the models are not the same. The variable explaining the largest percent of the variation for most volatiles (with the notable exception of terpinyl acetate + cadinene) was cultivar, which is interpreted as a pure genetic effect. The pure environment effect (year) was very large for only one of the volatiles (caryophyllene), but was moderate for three others. The year-by-cultivar interaction (representing the genetic-by-environment interaction) was moderate to large for four of the volatiles. Treatment effects (inoculated versus noninoculated and holding time) generally contributed less to the total variance, except for a relatively large holding time effect for terpinyl acetate + cadinene. The residual, representing plant-to-plant variation (and any other sources of model error), was relatively small $(16 \%$ or less), suggesting that within a cultivar, fruit from individual plants produced similar volatile concentrations.

CORRELATIONS A M ONG VOLATILES. The strongest correlations among volatiles occurred at the 0-d holding time, and lagged correlations among volatiles were lower than among those same volatiles from the same holding time (lagged results not shown). Table 4 presents correlations among volatiles at a holding time of $0 \mathrm{~d}$. Correlations among volatile extracts were generally similar in the 2 years despite data from fewer cultivars in 2005. The strongest correlations observed were those between the two aldehydes (for fruit extracts) and among the aldehydes and the terpene $\alpha$-terpineol for both extracts and whole fruit. Whole fruit volatiles had a different pattern of correlation from the extracts, especially for terpinyl acetate + cadinene. Although terpinyl acetate + cadinene usually behaved differently than the other volatiles in extracts, it was significantly correlated with trans-2-hexenal and the terpenes, caryophyllene and limonene. The only other significant correlation occurred between $\alpha$-terpineol and linalool for extracts in 2004 (there were insufficient cultivars to estimate the correlation in 2005).

\section{Discussion}

Anthracnose FRUIT ROT. Levels of anthracnose fruit rot in inoculated samples from this study were variable and did not necessarily follow expected results based on historical field experience and published data. For example, of the three major cultivars ('Duke', 'Elliott', and 'Bluecrop') grown in New Jersey, 'Duke' typically has low levels of anthracnose fruit rot (Draper et al., 1987) as does 'Elliott', whereas 'Bluecrop' can be quite high. In our inoculated samples from 2004, both 'Elliott' and 'Duke' had much higher levels (75\% and 53\%, respectively) of rot than expected, whereas the rot level in 'Bluecrop' was intermediate at $65 \%$. The reason for this discrepancy is unknown but may in part be attributable to different mechanisms of resistance expressed in the field (DeMarsay, 2005). Timing of exposure to inoculum may also be an important factor. We inoculated when the fruit were just beginning to ripen (judged by fruit color changes), whereas most published work, including Polashock et al. (2005), inoculated in the pea-sized green fruit stage. In 2005, levels of fruit rot were very high across all cultivars tested. Carryover of inoculum from 2004, because no fungicides were used, may have overwhelmed any cultivar resistance resulting in unusually high infection rates.

The usefulness of percent sample fruit rot in the multiple regression models is likely evidence of the underlying genetics 
Table 2. Significant effects for modeling ${ }^{z}$ volatile emissions from highbush blueberry. ${ }^{y}$

\begin{tabular}{|c|c|c|c|c|c|c|c|c|}
\hline $\begin{array}{l}\text { Independent variable }{ }^{\mathrm{x}} \\
\text { (Categorical or continuous) }\end{array}$ & Hexanal $^{\mathrm{w}}$ & $\begin{array}{l}\text { trans-2- } \\
\text { Hexenal }\end{array}$ & Caryophyllene & Limonene & $\begin{array}{l}\text { Terpinyl acetate } \\
+ \text { cadinene }\end{array}$ & Linalool & $\alpha$-Terpineol & $\alpha$-Farnesene \\
\hline Holding time & $\mathrm{S}^{\mathrm{v}}$ & $\mathrm{S}$ & & $\mathrm{S}$ & $\mathrm{S}$ & $\mathrm{S}$ & & \\
\hline Categorical, three levels & & $\begin{array}{l}S \\
S\end{array}$ & & $\mathrm{~S}$ & $\mathrm{~S}$ & & & \\
\hline
\end{tabular}

Treatment (inoculated or

noninoculated)

Categorical, two levels

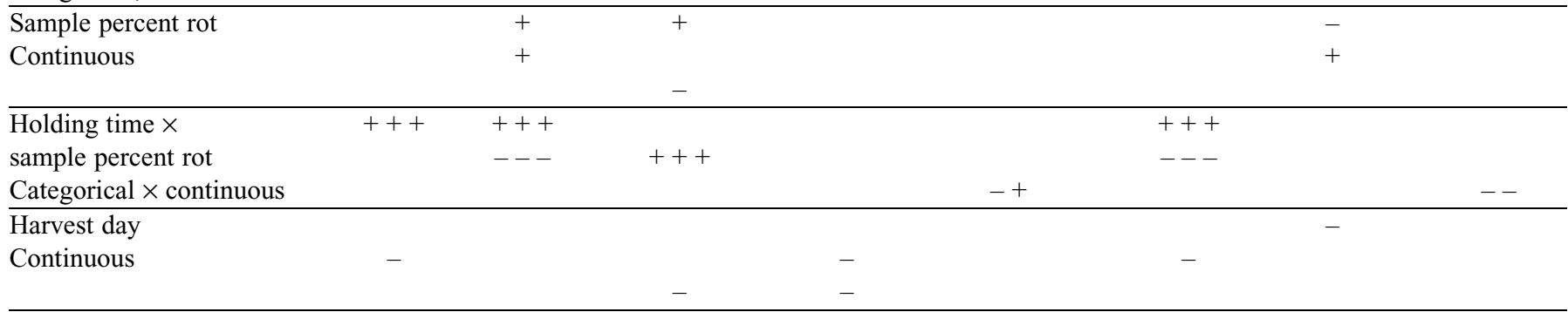

\section{Holding time $x$}

harvest day

Categorical $\times$ continuous

Percent rot $\times$ harvest day

Continuous

Date for $50 \%$ ripe

Continuous

$-$

\begin{tabular}{|c|c|c|c|c|c|c|c|c|}
\hline $\mathrm{R}^{2}$ for model & & 0.77 & 0.81 & 0.55 & 0.39 & 0.48 & 0.09 & 0.45 \\
\hline & 6 & 6 & 3 & 5 & 5 & 4 & 2 & \\
\hline & & 6 & 6 & 6 & 5 & 6 & 6 & 6 \\
\hline
\end{tabular}

${ }^{\mathrm{z}}$ Models were selected using Akaike's information criterion (AIC) in a stepwise procedure (significant $P$ values are from F tests, all significant $P \leq 0.01$, see text for details). Holding time and treatment (spraying with anthracnose spores) were design effects and were forced into all models; however, treatment was never significant.

${ }^{y}$ In-row order (under each volatile): 1) 2004 extracts, 2) 2005 extracts, 3) 2005 whole fruit with volatile concentration as the dependent variable. ${ }^{x}$ All significant independent variables from all modeling are shown. For each independent variable shown, significant effects, if any, are reported in row 1 for 2004 fruit extracts, in row 2 for 2005 fruit extracts, and in row 3 for 2005 whole fruit. Date for $50 \%$ ripe is based on 2004 field data.

${ }^{\text {wF }}$ or each volatile (other than for the main effect of holding time), significant effects are reported in columns for each holding time point (column one for $0 \mathrm{~d}$ extracts and 1 to $2 \mathrm{~d}$ whole fruit, column two for $2 \mathrm{~d}$ extracts and 5 to $6 \mathrm{~d}$ whole fruit, and column three for $4 \mathrm{~d}$ extracts).

"The letter "S" indicates a significant effect for holding time, and significant effects for covariates in the model are denoted by "+ " or "-_" depending on the sign of their coefficients.

of the various cultivars (affecting both fruit rot and volatile production) rather than volatile production being directly affected by the plant responding to the proportion of its berries affected by fruit rot. Because cultivar effects were not candidate independent variables in the model selection process, percent fruit rot would be one of the variables (the others are the two ripening day variables) that could substitute in part for a cultivar effect.

Volatile profiles. The cultivars used in this study exhibited dramatic differences in volatile profiles. The cultivar 'Duke' had high concentrations of all classes of volatiles, as previously noted (N. Vorsa, personal communication). Volatile profiles did not appear to be a function of cultivar species ancestry (e.g., 'Legacy' has the most complex ancestry among the cultivars tested but had one of the simplest volatile profiles; Table 1) nor does it appear, in this limited sample, that species ancestry is a predictor of fruit rot susceptibility (Table 1). This may be attributable in part to the selection of offspring from crosses with desirable aroma and flavor characteristics, both of which are impacted by fruit volatiles. Thus, volatile complexity and species-specific volatiles that may contribute to disease resistance, after an interspecific cross, may be lost after selection by the breeder for various horticultural traits and sensory characteristics.

Many factors affect the concentration of volatiles emitted from whole fruit and fruit extracts, including cultivar, fruit ripeness and processing methods, volatile collection methods, and so on (Forney, 2001). Volatiles such as aldehydes that increase as a result of wounding (Archbold et al., 1997; Hatanaka, 1993) are naturally much higher in fruit extracts than in whole fruit. Although volatiles from fruit extracts may be an indicator of potential production of certain compounds 
Table 3. Variance decomposition for volatiles from highbush blueberry extracts, given as percentage, using the same model for all volatiles.

\begin{tabular}{|c|c|c|c|c|c|c|c|}
\hline & Hexanal & trans-2-Hexenal & Caryophyllene & Limonene & $\begin{array}{c}\text { Terpinyl acetate }+ \\
\text { cadinene }\end{array}$ & Linalool & $\alpha$-Terpineol \\
\hline Treatment & 0 & 0 & 0 & 0 & 1 & 0 & 0 \\
\hline Cultivar $\times$ treatment & 2 & 1 & 0 & 0 & 2 & 0 & 0 \\
\hline Cultivar $\times$ holding time & 6 & 14 & 5 & 0 & 5 & 2 & 1 \\
\hline Treatment $\times$ holding time & 0 & 0 & 0 & 0 & 0 & 0 & 0 \\
\hline Year $\times$ treatment & 0 & 0 & 0 & 0 & 1 & 0 & 0 \\
\hline Residual & 16 & 16 & 11 & 3 & 14 & 8 & 9 \\
\hline
\end{tabular}

Table 4. Pearson correlations between mean highbush blueberry cultivar volatiles at holding time 0 d. ${ }^{\mathrm{z}}$

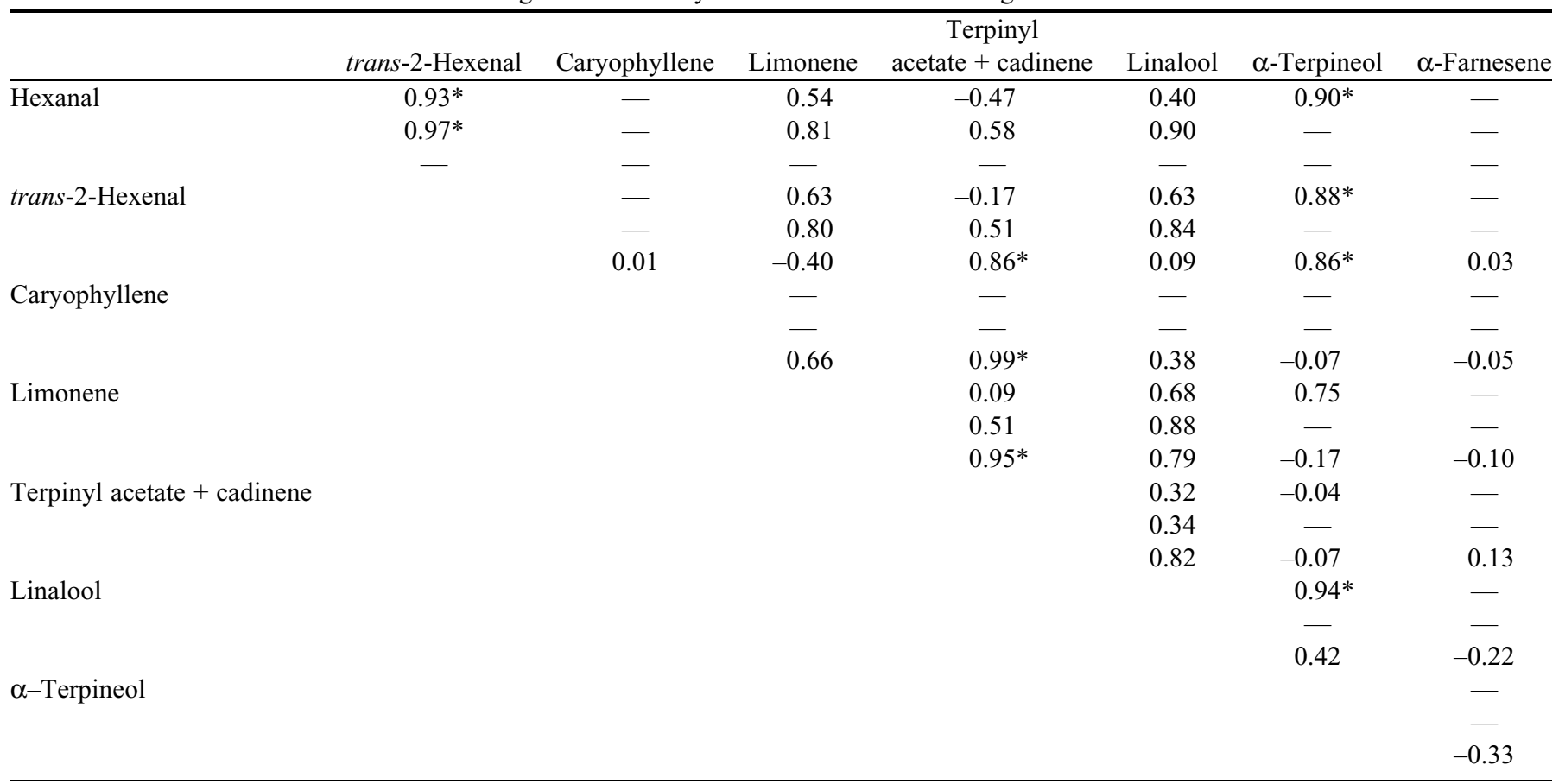

In-row order: 1) 2004 extracts, 2) 2005 extracts, 3) 2005 whole fruit. Correlations were calculated if more than three cultivar means were available. Significant correlations $(P<0.05)$ are denoted with an asterisk $(*)$.

such as trans-2-hexenal, it may also give a false impression of the concentrations typically encountered by a pathogen. However, C. acutatum infection causes small wounds as the spores germinate on the fruit surface and penetrate the epidermis, which may induce localized increases in emission of these compounds. In this study, background levels of anthracnose fruit rot, even in 2004, may have obscured differences between inoculated and noninoculated fruit. It is also possible that the "wounding" typically caused by this pathogen was smaller than that necessary to induce antimicrobial volatile emission.

Whole fruit volatile emission may be a better indicator of the antimicrobial status of the fruit because crushing fruit for extract preparation leads to cell damage-induced volatile production that could mask constitutive and induced antimicrobial volatile production. However, despite cultivar differences, we found no relationship between whole fruit volatile emissions and reported levels of anthracnose fruit rot resistance in highbush blueberry.

Although the inoculation treatment did not seem to trigger any changes in volatiles produced based on results from the stepwise regression analysis, there still appeared to be large environmental effects based on the variance decomposition that affect volatile production. These environmental effects are likely to be more weather-related (e.g., spring temperature and rainfall patterns) rather than the result of the presence or absence of specific pathogens at certain times of the year. Most of the variance seen in the volatile data can be attributed to genetic and environmental influences, not experimental manipulations. These results on fruit volatiles complement those on anthracnose resistance (Polashock et al., 2005), which showed there were large cultivar differences but also considerable yearto-year variability within cultivars.

Genotypes of maize (Zea mays L.) that were more resistant to Aspergillus flavus Link:Fr. were found to produce higher concentrations of $\mathrm{C}_{6}-\mathrm{C}_{12}$ aldehydes than the more susceptible genotypes (Zeringue et al., 1996), but a similar correlation between the concentrations of $\mathrm{C}_{6}$ aldehydes and anthracnose susceptibility in highbush blueberry was not detected. Conversely, some antifungal volatiles at low concentrations may 
play a role in promoting infection and diseases by certain fungal pathogens (Anderson et al., 1994; French, 1992; HamiltonKemp et al., 1992). French (1992), in numerous studies on fungal pathogens, showed that several volatile compounds can stimulate or inhibit spore germination and growth depending on concentration and treatment duration. A number of saturated volatiles, including hexanal, stimulated hyphal growth of Alternaria alternata (Fr.:Fr.) Keissl. and Botrytis cinerea Pers.:Fr. when supplied at low concentrations (Anderson et al., 1994; Hamilton-Kemp et al., 1992). Neither the cause for such stimulations nor the involvement, if any, of volatiles in promoting anthracnose fruit rot of highbush blueberry fruit is known.

There was no clear relationship across all highbush blueberry cultivars studied for volatiles emitted (both quantitative and qualitative differences) and resistance to anthracnose fruit rot. Except for 8-hydroxylinalool, all of the aldehydes and terpenes identified as evolving from the highbush blueberry samples in this study have been reported to have antifungal properties, although not necessarily against C. acutatum (Archbold et al., 1997; Azaz et al., 2002; Carson and Riley, 1995; Cavaleiro et al., 2006; Corbo et al., 2000; Fraternale et al., 2004; Govinden-Soulange et al., 2004; Himejima et al., 1992; Lanciotti et al., 1999; Peana et al., 1999; Plotto et al., 2003; Raintree Nutrition Inc., 2004; Song et al., 2007; Tabanca et al., 2006, 2007; Tepe et al., 2004; Wilson et al., 1997). Thus, the compounds we identified, although not correlated with anthracnose fruit rot resistance, may play a role in resistance to other highbush blueberry fruit diseases. Alternatively, additive or synergistic effects of complex mixtures of these and other phytochemicals, instead of a single compound or class of compounds, might provide antimicrobial benefits (Delaquis et al., 2002; Lambert et al., 2001). Some of these compounds may also be important for fungistatic effects in green fruit when field infection typically occurs. The fact that ripe fruit, even from "resistant" cultivars, are very susceptible to anthracnose infection and fruit rot (Fig. 3) suggests that cultivar resistances to this particular disease may be limited to qualitative and quantitative differences in antimicrobial volatiles during only the early stages of fruit development. Future studies should be directed at qualitative and quantitative volatile profiles throughout development from the green fruit stage to fully ripe.

\section{Literature Cited}

Al-Howiriny, T.A. 2003. Composition and antimicrobial activity of the essential oil of Salvia lanigera. Pakistan J. Biol. Sci. 6:133-135. Anderson, R.A., T.R. Hamilton-Kemp, D.F. Hildebrand, C.T. McCracken, Jr., R.W. Collins, and P.D. Fleming. 1994. Structureantifungal activity relationships among volatile C6 and C9 aliphatic aldehydes, ketones, and alcohols. J. Agr. Food Chem. 42:1563-1568. Archbold, D.D., T.R. Hamilton-Kemp, M.M. Barth, and B.E. Langlois. 1997. Identifying natural volatile compounds that control gray mold (Botrytis cinerea) during postharvest storage of strawberry, blackberry, and grape. J. Agr. Food Chem. 45:4032-4037.

Azaz, D., F. Demirci, F. Satil, M. Kurkcuoglu, and K.H.C. Baser. 2002. Antimicrobial activity of some Satureja essential oils. Zeitschrift fur Naturforschung 57c:817-821.

Baloga, D.W., N. Vorsa, and L. Lawter. 1995. Dynamic headspace gas chromatography-mass spectrometry analysis of volatile compounds from wild diploid blueberry species, p. 235-247. In: R.L. Rouseff and M.M. Leahy (eds.). Fruit flavors. Amer. Chem. Soc., Chicago.

Bate, N.J. and S.J. Rothstein. 1998. C6-volatiles derived from the lipoxygenase pathway induce a subset of defense-related genes. Plant J. 16:561-569.
Burnham, K.P. and D.A. Anderson. 1998. Model selection and inference: A practical information-theoretic approach. SpringerVerlag, New York.

Buttery, R.G. and L.C. Ling. 1993. Volatile components of tomato fruit and plant parts: Relationship and biogenesis, p. 23-34. In: R. Teranishi, R. Buttery, and H. Sugisawa (eds.). Bioactive volatile compounds from plants. Amer. Chem. Soc., Washington, DC.

Caccioni, D.R.L. and M. Guizzardi. 1994. Inhibition of germination and growth of fruit and vegetable post-harvest pathogenic fungi by essential oil components. J. Essential Oil Res. 6:173-179.

Carson, C.F. and T.V. Riley. 1995. Antimicrobial activity of the major components of the essential oil of Melaleuca alternifolia. J. Appl. Bacteriol. 78:264-269.

Cavaleiro, C., E. Pinto, M.J. Goncalves, and L. Salgueiro. 2006. Antifungal activity of Juniperus essential oils against dematophyte, Aspergillus and Candida strains. J. Appl. Microbiol. 100:1333-1338.

Chappell, J. 1995. The biochemistry and molecular biology of isoprenoid metabolism. Plant Physiol. 107:1-6.

Corbo, M.R., R. Lanciotti, F. Gardini, M. Sinigaglia, and M.E. Guerzoni. 2000. Effects of hexanal, trans-2-hexenal, and storage temperature on shelf life of fresh sliced apples. J. Agr. Food Chem. 48:2401-2408.

Cowan, M.M. 1999. Plant products as antimicrobial agents. Clin. Microbiol. Rev. 12:564-582.

Daykin, M.E. and R.D. Milholland. 1984. Infection of blueberry fruit by Colletotrichum gloeosporioides. Plant Dis. 68:948-950.

Delaquis, P.J., K. Stanich, B. Girard, and G. Mazza. 2002. Antimicrobial activity of individual and mixed fractions of dill, cilantro, coriander and eucalyptus essential oils. Int. J. Food Microbiol. 74:101-109.

DeMarsay, A. 2005. Anthracnose fruit rot of highbush blueberry: Biology and epidemiology. Rutgers, The State University of New Jersey, New Brunswick. PhD Diss.

Demirci, B., K.H. Baser, N. Tabanca, and D.E. Wedge. 2006. Characterization of volatile constituents of Haplopappus greenei and studies of the antifungal activity against phytopathogens. J. Agr. Food Chem. 54:3146-3150.

Ding, C.K., C.Y. Wang, K.C. Gross, and D.L. Smith. 2002. Jasmonate and salicylate induce the expression of pathogenesis-related protein genes and increase resistance to chilling injury in tomato fruit. Planta 214:895-901.

Dorman, H.J.D. and S.G. Deans. 2000. Antimicrobial agents from plants: Antibacterial activity of plant volatile oils. J. Appl. Microbiol. 88:308-316

Draper, A., G. Galletta, G. Jelenkovic, and N. Vorsa. 1987. 'Duke' highbush blueberry. HortScience 22:320 (abstr.).

Dudareva, N.D., E. Pichersky, and J. Gershenzon. 2004. Biochemistry of plant volatiles. Plant Physiol. 135:1893-1902.

Forney, C.F. 2001. Horticultural and other factors affecting aroma volatile composition of small fruit. HortTechnology 11: $529-538$.

Fraternale, D., D. Ricci, F. Epifano, and M. Curini. 2004. Compositional and antifungal activity of two essential oils of Hyssop (Hyssopus officinalis L.). J. Essential Oil Res. 16:617-622.

French, R.C. 1992. Volatile chemical germination stimulators of rusts and other fungal spores. Mycologia 84:277-288.

Gardini, F., R. Lanciotti, and M.E. Guerzoni. 2001. Effect of trans-2hexenal on the growth of Aspergillus flavus in relation to its concentration, temperature and water activity. Lett. Appl. Microbiol. $33: 50-55$.

Govinden-Soulange, J., N. Magan, A. Gurib-Fakim, A. Gauvin, J. Smadja, and H. Kodja. 2004. Chemical composition and in vitro antimicrobial activities of the essential oils from endemic Psiadia species growing in Mauritius. Biol. Pharmaceutical Bul. 27:18141818.

Hamilton-Kemp, T.R., C.T. McCrackin, Jr., J.H. Loughrin, R.A. Anderson, and D.F. Hildebrand. 1992. Effects of some natural volatile compounds on the pathogenic fungi Alternaria alternata and Botrytis cinerea. J. Chem. Ecol. 18:1083-1091. 
Hatanaka, A. 1993. The biogeneration of green odour by green leaves. Phytochemistry 34:1201-1218.

Himejima, M., K.R. Hobson, T. Otsuka, D.L. Wood, and I. Kubo. 1992. Antimicrobial terpenes from oleoresins of Ponderosa pine tree Pinus ponderosa: A defense mechanism against microbial invasion. J. Chem. Ecol. 18:1809-1818.

Knobloch, K., A. Pauli, B. Iberl, N. Weis, and H. Weigand. 1989. Antibacterial and antifungal properties of essential oil components. J. Essential Oil Res. 1:119-128.

Kramer, M. 2004. Automatic model selection in the mixed models framework. Proc. Kansas State Univ. Conf. Appl. Stat. Agr. 16:127140.

Kramer, M. 2005. R-squared statistics for mixed models. Proc. Kansas State Univ. Conf. Appl. Stat. Agr. 17:148-160.

Lambert, R.J.W., P.N. Skandamis, P.J. Coote, and G.-J.E. Nychas. 2001. A study of the minimum inhibitory concentration and mode of action of oregano essential oil, thymol and carvacrol. J. Appl. Microbiol. 91:453-462.

Lanciotti, R., M.R. Corbo, F. Gardini, M. Sinigaglia, and M.E. Guerzoni. 1999. Effect of hexanal on the shelf life of fresh apple slices. J. Agr. Food Chem. 47:4769-4776.

Latrasse, A. 1991. Fruits III, p. 329-388. In: H. Maarse (ed.). Volatile compounds in foods and beverages. Marcel-Dekker, New York.

Mari, M. and M. Guizzardi. 1998. The postharvest phase; emerging technologies for the control of fungal diseases. Phytoparasitica 26:59-66.

Milholland, R.D. 1995. Anthracnose fruit rot (ripe rot), p. 17. In: F.L. Caruso and D.C. Ramsdell (eds.). Compendium of blueberry and cranberry diseases. American Phytopathological Society, St. Paul, MN. Overton, S. and J.J. Manura. 1999. Volatile organic composition in blueberries. 27 Apr. 2006. <http://www.sisweb.com/referenc/applnote/ app-43.htm>.

Pawliszyn, J. 1997. Solid phase microextraction: Theory and practice. Wiley, New York.

Peana, A.T., M.D.L. Moretti, and C. Juliano. 1999. Chemical composition and antimicrobial action of the essential oils of Salvia desoleana and S. sclarea. Planta Med. 65:752-754.

Plotto, A., D.D. Roberts, and R.G. Roberts. 2003. Evaluation of plant essential oils as natural postharvest disease control of tomato (Lycopersicon esculentum). Acta Hort. 628:737-741.
Polashock, J.J., M.K. Ehlenfeldt, A.W. Stretch, and M. Kramer. 2005. Anthracnose fruit rot resistance in blueberry cultivars. Plant Dis. 89:33-38.

Raintree Nutrition Inc. 2004. Biological activities for compounds of jatoba (Hymenaea courbaril). 27 Apr. 2006. <http://www.raintree.com/jatoba-activity.pdf $>$.

Saftner, R.A. 1999. The potential of fruit coating and film treatments for improving the storage and shelf-life qualities of 'Gala' and 'Golden Delicious' apples. J. Amer. Soc. Hort. Sci. 124:682-689.

Saftner, R.A., J.A. Abbott, W.S. Conway, C.L. Barden, and B.T. Vinyard. 2002. Instrumental and sensory quality characteristics of 'Gala' apples in response to prestorage heat, controlled atmosphere, and air storage. J. Amer. Soc. Hort. Sci. 127:1006-1012.

Sipailiene, A., P.R. Venskutonis, R. Beranauskiene, and A. Sarkinas. 2006. Antimicrobial activity of commercial samples of thyme and marjoram oils. J. Essential Oil Res. 18:1-7.

Song, L., J.Y. Ding, C. Tang, and C.H. Yin. 2007. Compositions and biological activities of essential oils of Kadsura longepedunculata and Schisandra sphenanthera. Amer. J. Chin. Med. 35:353-364.

Tabanca, N., B. Demirci, K.H. Baser, Z. Aytac, M. Ekici, S.I. Khan, M.R. Jacob, and D.E. Wedge. 2006. Chemical composition and antifungal activity of Salvia macrochlamys and Salvia recognita essential oils. J. Agr. Food Chem. 54:6593-6597.

Tabanca, N., B. Demirci, K.H. Baser, E. Mincsovics, S.I. Khan, M.R. Jacobs, and D.E. Wedge. 2007. Characterization of volatile constituents of Scaligeria tripartite and studies on the antifungal activity against phytopathogenic fungi. J. Chromatogr. B 850:221-229.

Tepe, B., D. Daferera, M. Sökmen, M. Polissiou, and A. Sökmen. 2004. In vitro antimicrobial and antioxidant activities of the essential oils and various extracts of Thymus eigii M. Zohary et P.H. Davis. J. Agr. Food Chem. 52:1132-1137.

Utama, I.M.S., R.B.H. Wills, S. Ben-Yehoshua, and C. Kuek. 2002. In vitro efficacy of plant volatiles for inhibiting the growth of fruit and vegetable decay microorganisms. J. Agr. Food Chem. 50:6371-6377. Wilson, C.L., J.M. Solar, A. El Ghaouth, and M.E. Wisniewski. 1997. Rapid evaluation of plant extracts and essential oils for antifungal activity against Botrytis cinerea. Plant Dis. 81:204-210.

Zeringue, H.J., R.L. Brown, J.N. Neucere, and T.E. Cleveland. 1996. Relationships between C6-C12 alkanal and alkenal volatile contents and resistance of maize genotypes to Aspergillus flavus and aflatoxin production. J. Agr. Food Chem. 44:403-407. 